\title{
Co-expression network analysis of virulence genes exoS and exoU of pseudomonas aeruginosa in lower respiratory tract based on histological data expression profiles
}

\author{
Erli Jiao ${ }^{1}$, Bo Chen*2 \\ ${ }^{1}$ Clinical Laboratory, Baogang Hospital, Baotou, Inner Mongolia, China \\ ${ }^{2}$ The First Affiliated Hospital of Baotou Medical College, Inner Mongolia University of Science and Technology, Baotou, Inner \\ Mongolia, China
}

Received: March 10, 2020

DOI: $10.5430 /$ dec.v6n4p10
Accepted: May 21, 2020

Online Published: May 29, 2020

\begin{abstract}
Objective: To use the gene chip of pseudomonas aeruginosa as a research sample and to explore it at an omics level, aiming at elucidating the co-expression network characteristics of the virulence genes exoS and exoU of pseudomonas aeruginosa in the lower respiratory tract from the perspective of molecular biology and identifying its key regulatory genes.

Methods: From March 2016 to May 2018, 312 patients infected with pseudomonas aeruginosa in the lower respiratory tract who were admitted to Department of Respiratory Medicine of Baogang Hospital and given follow-up treatments in the hospital were selected as subjects by use of cluster sampling. Alveolar lavage fluid and sputum collected from those patients were used as biological specimens. The genes of pseudomonas aeruginosa were detected with the help of oligonucleotide probes to make a pre-processing of chip data. A total of 8 common antibiotics (ceftazidime, gentamicin, piperacillin, amikacin, ciprofloxacin, levofloxacin, doripenem and ticarcillin) against Gram-negative bacteria were selected to determine the drug resistance of biological specimens. MCODE algorithm was used to construct a co-expression network model of the drug-resistance genes focused on exoS/exoU.

Results: The expression level of exoS/exoU in the drug-resistance group was significantly higher than that in the non-resistance group $(p<.05)$. The top 5 differentially expressed genes in the alveolar lavage fluid specimens from the drug-resistance group were RAC1, ITGB1, ITGB5, CRK and IGF1R in the order from high to low. In the sputum specimens, the top 5 differentially expressed genes were RAC1, CRK, IGF1R, ITGB1 and ITGB5. In the alveolar lavage fluid specimens, only RAC1 had a positive correlation with the expression of exoS and exoU $(p<.05)$. In the sputum specimens, RAC1, ITGB1, ITGB5, CRK and IGF1R were positively correlated with the expression of exoS and exoU $(p<.05)$. The genes included in the co-expression network contained exoS, exoU, RAC1, ITGB1, ITGB5, CRK, CAMK2D, RHOA, FLNA, IGF1R, TGFBR2 and FOS. Among them, RAC1 had a highest score in the aspect of regulatory ability (72.00) and the largest number of regulatory genes (6); followed by ITGB1, ITGB5 and CRK genes.

Conclusions: The high expression of exoS and exoU in the sputum specimens suggests that pseudomonas aeruginosa has a higher probability to get resistant to antibiotics; RAC1, ITGB1, ITGB5 and CRK genes may be the key genes that can regulate the expression of exoS and exoU.
\end{abstract}

Key Words: Omics data expression profile, Lower respiratory tract, Pseudomonas aeruginosa, exoS, exoU, Co-expression network

*Correspondence: Bo Chen; Email: nmgbgyyyy@163.com; Address: The First Affiliated Hospital of Baotou Medical College, Inner Mongolia University of Science and Technology, Baotou, Inner Mongolia, 014010, China. 


\section{INTRODUCTION}

Pseudomonas aeruginosa is one of the most commonly seen drug-resistance bacteria in the lower respiratory tract infection. It is reported that ${ }^{[1-4]}$ the drug-resistance genes of pseudomonas aeruginosa mainly consist of some gene families such as mecA/aph/tetM/aac/ermA. In addition, there exist some differences in methylation level, copy number variation, carrying and combination mode as well as transcript of the drug-resistance genes, the high variation of which may be a decisive factor for the drug resistance of pseudomonas aeruginosa. From the perspective of clinical medication, it is indisputable that one of the most important missions is to enhance the rational use of antibiotics. It means that the preliminary antimicrobial susceptibility test is required no matter in the aspect of dosage or type. It is not only proposed to reduce the occurrence of nosocomial infection, but also objectively reduce the sensitivity of the drug-resistance genes of the corresponding strain. It is also feasible to establish a more rational medication pattern for patients with the combination of the particularity of drug interactions (such as synergistic effect, antagonistic effect, joint effect, independent effect, etc.). In the meantime, with the completion of the human genome project and the progress of clinical molecular diagnosis technology, the application of bioinformatics concerning the exploration of omics data gradually becomes a research topic of intense interest at present. ${ }^{[5-7]}$ Thereinto, the co-expression network is a method designed specifically to describe the relationship of gene interactions. Through different combination patterns of dots and lines, the diagram of the co-expression network can present the relationship of gene interactions and their weight in a very intuitive way. ${ }^{[5-7]}$ The genetic principle has thoroughly demonstrated that the molecular biological mechanism for the occurrence of drug resistance is the consequence of the synergistic (or antagonistic) effect of multiple genes. Hence, this study is designed to take gene chips as research samples and explore them at an omics level, aiming at elucidating the formation mechanism for drug resistance of virulence genes exoS and exoU of pseudomonas aeruginosa in the lower respiratory tract infection.

\section{DATA AND METHODS}

\subsection{General information}

From March 2016 to May 2018, 312 patients infected with pseudomonas aeruginosa in the lower respiratory tract who were admitted to Department of Respiratory Medicine of Baogang Hospital and given follow-up treatment in the hospital were selected as subjects by use of cluster sampling. After explaining the research purpose to the subjects, specimens (including sputum and alveolar lavage fluid, each of

Published by Sciedu Press them was equally divided in duplicate and placed under the temperature of $-20^{\circ} \mathrm{C}$ for different detections) were subsequently collected from these patients. The containers of sputum specimens should be kept clean, wide-mouthed, sterile, covered, sealed, leak-proof and disposable, and they can be acquired from Clinical Laboratory. For most of the subjects, the sputum volume should be largest in the morning, with a highest bacterial concentration. It was recommended for the patients to use gargle and then use cold boiled water or normal saline to remove bacteria from the oral cavity. Subsequently, each of them was instructed to expectorate one to two mouthfuls of sputum into a sterile jar after deep breath. For the patients with a small sputum volume, it was workable to use $45^{\circ} \mathrm{C} 10 \%$ sodium chloride solution to perform an atomization inhalation treatment to lead out sputum.

\subsection{Research methods}

\subsubsection{Inoculation}

The specimens were required to be inoculated in Columbia agar plates and MacConkey agar plates, and then cultured in the constant temperature incubator at $35^{\circ} \mathrm{C}$ for $24-48 \mathrm{~h}$ in order to isolate pseudomonas aeruginosa.

\subsubsection{The cultivation of pseudomonas aeruginosa in the lower respiratory tract infection and the amplifica- tion of the products}

The primers were designed according to the bioinformatics data of the related gene sequence in Genbank database in NCBI and synthesized by Genminix Biotechnology Co., Ltd.. The sequence of the upstream primer was: 5'- GTT CAG GTA GCG ATG AGC TAG CAA C-3', and the sequence of the downstream primer was: 5'-CTG CAT GCA GTG AGC AGA GAG CGT C-3'. The primer length was $592 \mathrm{bp}$, and the reaction temperature was as follows: $95^{\circ} \mathrm{C}, 60 \mathrm{~s} / 55^{\circ} \mathrm{C}$, $30 \mathrm{~s} / 75^{\circ} \mathrm{C}, 60 \mathrm{~s}$. After 40 circles, the primers were extended for $10 \mathrm{~min}$ at the temperature of $75^{\circ} \mathrm{C}$.

\subsubsection{The preparation of gene chips}

The genes of 312 strains of pseudomonas aeruginosa were detected with the help of oligonucleotide probes (Thermo Fisher Scientific Inc. (Beijing)) to make a pre-processing of chip data (to convert the acquired hybridization signals to the omics expression profile data). In this study, the method of expression profile data normalization was global normalization. ${ }^{[8]}$ Meanwhile, SAM (significance analysis of microarrays) algorithm ${ }^{[9]}$ was applied to the screening of differential genes, and Fold Change value was considered as a standard to judge whether there existed the differential expression of a certain gene. The method of calculation was as follows: 


$$
\text { Fold change }=\left\{\begin{array}{l}
\frac{2^{x 1}}{2^{x^{2}}}, \text { if } x 1 \geqslant x 2 \\
\frac{-2^{x^{2}}}{2^{x 1}}, \text { if } x 1<x 2
\end{array}\right.
$$

Whereas, $\mathrm{x} 1$ was set as the signal mean value of the specimens in the drug-resistance group (a total of 8 common antibiotics in Department of Respiratory Medicine were selected to make a drug-resistance analysis, and drug-resistance to no less than 3 types was defined as drug-resistance. Therefore, such specimens were included in the drug-resistance group), and $\mathrm{x} 2$ was set as the signal mean value of the specimens in the non-resistance group.

\subsubsection{The principle and process of co-expression network analysis}

The original information for the construction of coexpression network was based on the original samples (i.e., sputum or alveolar lavage fluid specimens acquired through biological sampling in this study). Nevertheless, in the process of analysis, it was workable to utilize various databases (KEGG/Reactome/BIND/Biogrid/HPRD) to make an indepth analysis (including the comparison with the results from other researches) of omics data (such as differential genes and proteins, microRNA target genes and copy number variation) in the specimens. The co-expression network was made up of multiple signaling pathways, and the genes were considered as nodes (the size of the nodes can determine the regulatory ability of the gene). The area of the nodes was positively correlated with the regulatory ability of the gene. MCODE algorithm was applied to the analysis of the regulatory weight of the gene in the whole network model. In essence, it was a weighting approach based on clustering coefficients. ${ }^{[10]}$

\subsection{Statistical treatment}

The comparison in the expression of exoS/exoU between the drug-resistance group and the non-resistance group was described by use of $\bar{X} \pm \mathrm{s}$ and $\mathrm{M}\left(\mathrm{P}_{25}-\mathrm{P}_{75}\right)$ in combination with data normality test results. The comparison between two groups was made by use of $t$ test and $Z$ test. Similarly, the analysis on the correlation of differentially expressed genes with exoS/exoU was made by use of linear correlation and rank correlation. The difference $(p<.05)$ was of statistical significance.

\section{RESUlts}

\subsection{Analysis on the correlation of the expression of viru- lence genes $\operatorname{exoS}$ and $\operatorname{exo} U$ with the drug resistance of pseudomonas aeruginosa in the lower respiratory tract infection}

A total of 8 common antibiotics (ceftazidime, gentamicin, piperacillin, amikacin, ciprofloxacin, levofloxacin, doripenem and ticarcillin) in Department of Respiratory Medicine against Gram-negative bacteria were selected to consolidate and analyze the analysis results of the gene chips of sputum and alveolar lavage fluid specimens. The results showed that the expression of exoS/exoU in the drugresistance group was significantly higher than that in the non-resistance group, and the difference was of statistical significance $(p<.05)$. See Table 1 for details.

Table 1. Analysis on the correlation of the expression of exoS and exoU with the drug resistance of pseudomonas aeruginosa in the lower respiratory tract infection

\begin{tabular}{|c|c|c|c|c|c|c|c|c|}
\hline Item & Ceftazidime & Gentamicin & Piperacillin & Amikacin & Ciprofloxacin & Levofloxacin & Doripenem & Ticarcillin \\
\hline Drug resistance (\%) & $\begin{array}{l}19.32 \\
(85 / 440)\end{array}$ & $\begin{array}{l}3.18 \\
(14 / 440)\end{array}$ & $\begin{array}{l}11.36 \\
(49 / 440)\end{array}$ & $\begin{array}{l}21.82 \\
(96 / 440)\end{array}$ & $\begin{array}{l}29.32 \\
(129 / 440)\end{array}$ & $\begin{array}{l}8.18 \\
(36 / 440)\end{array}$ & $\begin{array}{l}2.50 \\
(11 / 440)\end{array}$ & $\begin{array}{l}7.27 \\
(32 / 440)\end{array}$ \\
\hline $\begin{array}{l}\text { Expression level of } \\
\text { exoS in the } \\
\text { drug-resistance group }\end{array}$ & $5.03 \pm 0.92$ & $4.32 \pm 0.86$ & $3.70 \pm 0.72$ & $\begin{array}{l}3.94 \\
(2.95,4.90)\end{array}$ & $3.82 \pm 0.75$ & $3.90 \pm 0.71$ & $\begin{array}{l}3.85 \\
(3.44,4.20)\end{array}$ & $3.59 \pm 0.88$ \\
\hline $\begin{array}{l}\text { Expression level of } \\
\text { exoS in the } \\
\text { non-resistance group }\end{array}$ & $1.21 \pm 0.57$ & $1.02 \pm 0.34$ & $1.66 \pm 0.39$ & $\begin{array}{l}1.80 \\
(1.54,2.21)\end{array}$ & $1.47 \pm 0.44$ & $1.52 \pm 0.32$ & $\begin{array}{l}1.67 \\
(1.44,2.13)\end{array}$ & $1.83 \pm 0.63$ \\
\hline$t_{1} / Z_{1}$ & 13.362 & 14.455 & 7.150 & 3.696 & 8.663 & 8.483 & 4.839 & 3.415 \\
\hline$p_{1}$ & $<.001$ & $<.001$ & $<.001$ & $<.001$ & $<.001$ & $<.001$ & $<.001$ & $<.001$ \\
\hline $\begin{array}{l}\text { Expression level of } \\
\text { exoU in the } \\
\text { drug-resistance group }\end{array}$ & $4.94 \pm 1.03$ & $4.77 \pm 1.22$ & $4.91 \pm 1.79$ & $\begin{array}{l}4.82 \\
(4.15,5.35)\end{array}$ & $4.67 \pm 1.32$ & $4.92 \pm 1.16$ & $\begin{array}{l}4.98 \\
(4.56,5.33)\end{array}$ & $4.60 \pm 1.34$ \\
\hline$t_{2} / Z_{2}$ & 8.182 & 6.838 & 9.211 & 6.199 & 5.356 & 7.570 & 6.757 & 4.955 \\
\hline$p_{2}$ & $<.001$ & $<.001$ & $<.001$ & $<.001$ & $<.001$ & $<.001$ & $<.001$ & $<.001$ \\
\hline
\end{tabular}




\subsection{The correlation of the top 5 differentially expressed genes in the drug-resistance group with the expres- sion of virulence genes exoS and exoU}

The top 5 differentially expressed genes in the alveolar lavage fluid specimens from the drug-resistance group were RAC1, ITGB1, ITGB5, CRK and IGF1R in the order from high to low. In the sputum specimens, the top 5 differentially expressed genes were RAC1, CRK, IGF1R, ITGB1 and ITGB5. In the alveolar lavage fluid specimens, only RAC1 had a positive correlation with the expression of exoS and exoU $(p<$ .05). In the sputum specimens, RAC1, ITGB1, ITGB5, CRK and IGF1R were positively correlated with the expression of exoS and exoU $(p<.05)$. See Tables 2 and 3.

Table 2. The correlation of the top 5 differentially expressed genes in the alveolar lavage fluid specimens from the drug-resistance group with the expression of virulence genes exoS and exoU

\begin{tabular}{llllll}
\hline Item & RAC1 & CRK & IGF1R & ITGB1 & ITGB5 \\
\hline exoS & $0.335^{*}$ & 0.026 & 0.039 & 0.074 & 0.077 \\
exoU & $0.285^{*}$ & 0.113 & 0.082 & 0.102 & 0.040 \\
\hline Note. ${ }^{*} p<.05$.
\end{tabular}

Table 3. The correlation of the top 5 differentially expressed genes in the sputum specimens from the drug-resistance group with the expression of virulence genes exoS and exoU

\begin{tabular}{llllll}
\hline Item & RAC1 & ITGB1 & ITGB5 & CRK & IGF1R \\
\hline exoS & $0.328^{*}$ & $0.372^{*}$ & $0.270^{*}$ & $0.336^{*}$ & $0.392^{*}$ \\
exoU & $0.447^{*}$ & $0.321^{*}$ & $0.306^{*}$ & $0.317^{*}$ & $0.420^{*}$ \\
\hline Note. ${ }^{*} p<.05$ & & & &
\end{tabular}

The main function of RAC1 encoding protein is to activate the signaling pathway of 1-phosphatidylinositol 3-kinase (PI3K)-Akt, control the dynamic balance of the cell quantity in local tissues and participate in cytoskeleton reassembly, and its variation characteristic is up-regulation; the main function of ITGB1 encoding protein is to participate in cell adhesion, recognize membrane receptors and improve protein kinase activity, and its variation characteristic is upregulation; the main function of ITGB5 encoding protein is to participate in hemostasis, vascular remodeling, immune response, MHC I antigen processing and peptide antigen submission, and the variation characteristic is up-regulation; the main function of CRK encoding protein is to be related with adaptive immune response and answer the activation of TGF- $\beta$ signaling pathway, and the variation characteristic is up-regulation; the main function of IGF1R encoding protein is to participate in the negative regulating process of the signaling pathway of TGF- $\beta$, this type of protein can work as an anti-apoptotic agent in most of malignant tissues to improve the survival rate of cells, and its variation characteristic is Published by Sciedu Press up-regulation.

\subsection{The construction of co-expression network model of drug-resistance determining genes focused on exoS/exoU}

The genes included in the co-expression network in this study were exoS, exoU, RAC1, ITGB1, ITGB5, CRK, CAMK2D, RHOA, FLNA, IGF1R, TGFBR2 and FOS, and exoS/exoU was considered as the core to construct the co-expression network model of drug-resistance determining genes of pseudomonas aeruginosa. Among them, RAC1 had a highest score in the aspect of regulatory ability (72.00) and the largest number of regulatory genes (8); followed by ITGB1, ITGB5 and CRK genes. See Figure 1 for the co-expression network model of drug-resistance determining genes focused on exoS/exoU, and see Table 4 for the score of the regulatory ability of each gene and the number of regulatory genes.

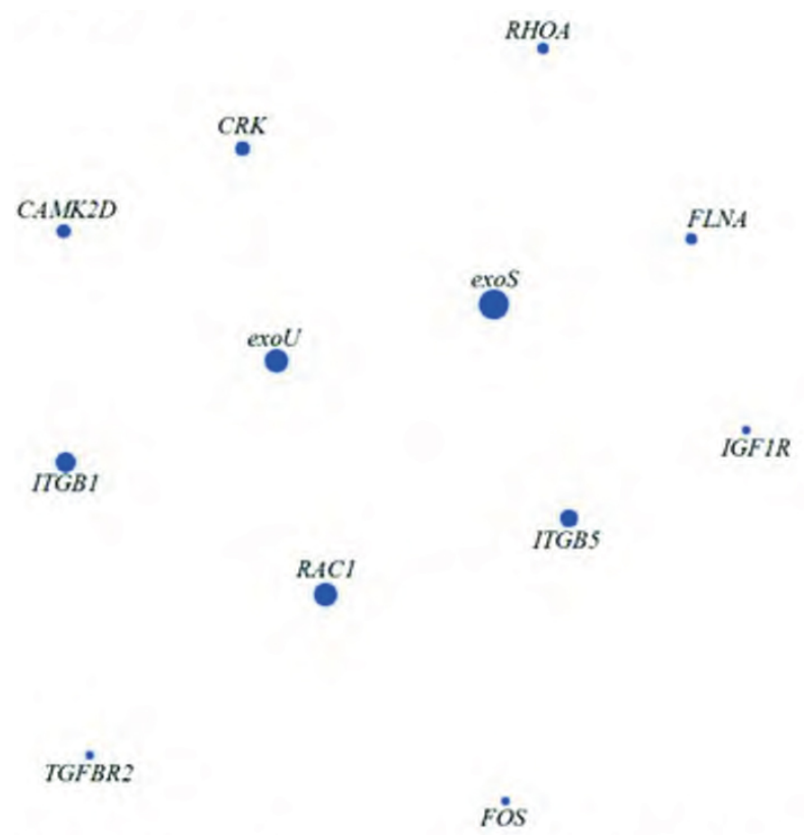

Figure 1. Co-expression network model of drug-resistance determining genes focused on exoS/exoU

\section{Discussion}

It is found in this research that, for any selected antibiotic, there is a statistically significant difference in the expression of exoS/exoU no matter in the drug-resistance group or the non-resistance group. It is indicated that exoS/exoU is highly correlated to the formation of the drug-resistance mechanism of pseudomonas aeruginosa, which is similar with the results from the same type of research at home and abroad. According to the past reports, the drug-resistance mechanism of virulence gene exoS/exoU involves multiple aspects: (1) the generation of inactivated enzymes (such as $\beta$ - 
lactamases, aminoglycosides modifying enzymes, and the generation of the former is the main mechanism of drug resistance); (2) lowered membrane permeability: many glycoproteins in the membrane of pseudomonas aeruginosa can actively exclude antibiotics (the exclusion systems contain MexAB-oprM, MexCD-oprJ, MexEF-oprN, MexJk-oprM, MexGHI-opmD, Mex-VW-oprM and MexXY-oprM), reduce the permeability of bacterial outer membrane and generate the synergistic effect of drug resistance. ${ }^{[13-15]}$

Table 4. Score of the regulatory ability of each gene and the number of regulatory genes in the co-expression network model

\begin{tabular}{lll}
\hline $\begin{array}{l}\text { Name of } \\
\text { Gene }\end{array}$ & $\begin{array}{l}\text { Score of the } \\
\text { regulatory ability }\end{array}$ & $\begin{array}{l}\text { The number of } \\
\text { regulatory genes }\end{array}$ \\
\hline exoS & 127.33 & 8 \\
exoU & 75.33 & 6 \\
RAC1 & 72.00 & 6 \\
ITGB1 & 64.00 & 5 \\
ITGB5 & 51.00 & 4 \\
CRK & 36.67 & 3 \\
CAMK2D & 33.00 & 3 \\
RHOA & 22.67 & 2 \\
FLNA & 22.33 & 2 \\
IGF1R & 3.67 & 1 \\
TGFBR2 & 3.67 & 1 \\
FOS & 3.00 & 1 \\
\hline
\end{tabular}

The genes included in the co-expression network in this study include exoS, exoU, RAC1, ITGB1, ITGB5, CRK, CAMK2D, RHOA, FLNA, IGF1R, TGFBR2 and FOS. In the co-expression network model of drug-resistance genes focused on exoS/exoU, RAC1 had a highest score in the aspect of regulatory ability (72.00), followed by ITGB1, ITGB5 and CRK. It may be closely associated with the drug resistance of exoS and exoU, and it is probable to be the key gene for regulating the expression of exoS/exoU. It has been found in the research that, ${ }^{[15]}$ the encoding products of RAC1 can lead to gene mutation of two types of topoisomerase, which is associated with the formation of the drug-resistance mechanism of pseudomonas aeruginosa. Gene mutation may prevent antibiotics from stably binding to enzyme-DNA complexes, so that the anti-bacterial effect is lost consequently. In addition, as ITGB gene family has a protective effect in the biofilm (polysaccharide matrix, fibrous proteins and other complexes secreted by bacteria after adhesion to and proliferation on the surface of inert matters) of pseudomonas aeruginosa, it can prevent DNA topoisomerase II and IV of pseudomonas aeruginosa from being inactivated and avoid organism immunity to a certain degree. Nevertheless, CRK is associated with the structural variation of integron, which is a type of gene-captured genetic unit existing on bacterial plasmids and chromosomes. They can transfer between bacteria by conjugation, transformation, transduction and transposition, which becomes an important factor for the rapid development of the drug resistance of pseudomonas aeruginosa. It is more obvious in Gram-negative bacteria, particularly in pseudomonas aeruginosa. The biofilm of pseudomonas aeruginosa can generate a large amount of granulocyte colony-stimulating factors, while IGF1 mediates in this signaling process; at the same time, IGF1 can also induce exoS and exoU genes to participate in the interaction of epithelium and mesenchyme. It is thus found that, as a typical representative of Gramnegative bacteria, pseudomonas aeruginosa has a great difference in the drug-resistance gene from Gram-positive bacteria. Moreover, the loss of membrane microporous protein genes ITGB1 and ITGB5 and the generation of carbapenaemse may have a certain synergistic effect with this pathway. During the process of the corresponding messenger playing a role in the synergistic effect, microporous proteins are influenced by promoter genes or upstream genes, and this influence is manifested as the up-regulation (the drug-resistance mechanism for most of bacteria) or down-regulation of the activity of encoding protein, so that the reason for the occurrence of drug resistance is explained from the perspective of cytobiology. ${ }^{[12]}$

In this study, it is found that the co-expression network analysis of exoS and exoU based on omics data expression profile has the following advantages in the process of study on the formation of the drug-resistance mechanism: (1) the omics data in the biological specimens are thoroughly used to explore the genetic data of genes and protein products of pseudomonas aeruginosa in different biological specimens (sputum and alveolar lavage fluid) from all patients with the help of high-density gene probes, make a comparison of the research results in combination with the omics information such as KEGG, Bingo and Gene Ontology data, and provide many molecular biological demonstrations for the formation of the drug-resistance mechanism of pseudomonas aeruginosa; (2) the co-expression network model makes a quantitative analysis of gene interactions, with MCODE algorithm used to maximize the utilization of the genetic information of pseudomonas aeruginosa (Fluorescent staining signal values in the gene chips are used to completely quantify the research results in combination with the changing rule of different gene detection sites in the same chip and recover the contribution weight coefficients of genes to drug resistance), providing a theoretical foundation for the follow-up 
in-vivo and in-vitro tests on the function of genes and protein products; (3) with the application of weight evaluation model, the co-expression network model can directly reflect the primary and secondary relation and the research value of gene action to further support the previously acquired research conclusions.

It is found in the recent related literatures that, ${ }^{[1-4,11-15]}$ the drug resistance of pseudomonas aeruginosa is mostly caused by the gene mutation of its own. In nature, this type of mutation can be manifested as a self-defense mechanism of bacteria, i.e., bacteria can withstand various adverse factors in the environment by encoding the corresponding proteins. Meanwhile, abundant evidence confirms that the drug resistance of pseudomonas aeruginosa doesn't result from a single gene, but is caused by various genes and their interactions. It can also get resistant to antibiotics by consolidating its encoding proteins at a functional level. It can also indicate that to explore the function, mechanism and signaling pathway of the key genes which can lead to drug resistance is a subsequent research focus for bacterial drug resistance.

It is noted that the biological effectiveness dose may be also an important factor for the determination of bacterial drug resistance. MIC0, MIC50, MIC90, MIC100, ID0, ID50, ID90 and ID100 are indicators which can directly reflect drug dose and the corresponding toxicological relationship, and they are considered as a significant factor for the drug resistance of the same individual to the same drug. However, the steps in the corresponding toxicological analysis of drugs are rarely applied directly in the clinic, the potential effects are often ignored. ${ }^{[16,17]}$

\section{Conclusion}

Taking the exploration of omics data as the research basis, this study is intended to construct the co-expression network model of virulence genes exoS and exoU of pseudomonas aeruginosa in the respiratory tract, analyze the action pattern of the key genes and provide a new idea for the study on the drug-resistance mechanism of pseudomonas aeruginosa in the respiratory tract.

\section{CONFlicts of InTEREST Disclosure}

The authors declare they have no conflicts of interest.

\section{REFERENCES}

[1] Saeed M, Rasheed F, Afzal RK, et al. Pseudomonas aeruginosa: Evaluation of Pathogen Burden and Drug-Resistance Trends in a Tertiary Care Hospital. J Coll Physicians Surg Pak. 2018; 28(4): 279. PMid:29615167. https://doi.org/10.29271/jcpsp. 2018.04 .279

[2] Elshouny WA, Ali SS, Sun J, et al. Drug resistance profile and molecular characterization of extended spectrum beta-lactamase $(\mathrm{ES} \beta \mathrm{L}$ )-producing Pseudomonas aeruginosa isolated from burn wound infections. Essential oils and their potential for utilization. Microb Pathog. 2018; 116(3): 301-312. PMid:29407236. https: //doi.org/10.1016/j.micpath.2018.02.005

[3] Si WX, Zhang ZJ, Sun JN, et al. Distribution and changes of drug resistance of Pseudomonas aeruginosa from 2013 to 2017. Chinese Journal of Nosocomiology. 2018; 28(24): 3705-3708.

[4] Guo N, Zhang N, Yan L. Weighted gene co-expression network analysis in identification of key genes and networks for ischemicreperfusion remodeling myocardium. Mol Med Rep. 2018; 18(2): 1955-1962. PMid:29901145. https://doi.org/10.3892/mmr. 2018.9161

[5] Liu BH. Differential Coexpression network analysis for gene expression data. Methods Mol Biol. 2018; 1754: 155. PMid:29536442. https://doi.org/10.1007/978-1-4939-7717-8_9

[6] Ferrari C, Proost S, Ruprecht C, et al. Phyto-Net: comparative co-expression network analyses across phytoplankton and land plants. Nucleic Acids Res. 2018; 46(W 1): 76-83. PMid:29718316. https://doi.org/10.1093/nar/gky298

[7] Mortensen KN, Gjedde A, Thompson GJ, et al. Impact of Global Mean Normalization on Regional Glucose Metabolism in the Hu- man Brain. Neural Plast. 2018; 12(10): 1-16. PMid:30008742. https://doi.org/10.1155/2018/6120925

[8] Sun A, Zhan B, Li M, et al. Screening Small Metabolites from Cells as Multifunctional Coatings Simultaneously Improves Nanomaterial Biocompatibility and Functionality. Adv Sci (Weinh). 2018; 5(7): 1800341. PMid:30027060. https://doi.org/10.1002/advs.2 01800341

[9] Maschietto M, Tahira AC, Puga R, et al. Co-expression network of neural-differentiation genes shows specific pattern in schizophrenia. Bmc Med Genomics. 2015; 8(1): 23. PMid:25981335. https : //doi.org/10.1186/s12920-015-0098-9

[10] Kainuma A, Momiyama K, Kimura T, et al. An outbreak of fluoroquinolone-resistant Pseudomonas aeruginosa ST357 harboring the exoU gene. J Infect Chemother. 2018; 24(8): 615 . PMid:29628388. https://doi.org/10.1016/j.jiac.2018.03 .008

[11] Wang J, Liang YZ, Dai PJ, et al. Detection of type three secretion system virulence gene expression difference of pseudomonas aeruginosa in Zhongshan area. International Journal of Laboratory Medicine. 2017; 38(6): 801-804.

[12] Ju XH, Li Y, Wang YH, et al. Correlation between virulence genotype and fluoroquinolone drugs resistance in Pseudomonas aeruginosa of lower respiratory tract infection. Chinese Journal of Zoonoses. 2017; 33(1): $38-42$

[13] Ju XH, Li Y, Wang YH. Distribution and drug-resistance of virulence gene exoS and exoU in Pseudomonas aeruginosa. Chinese Journal of Hospital Pharmacy. 2017; 37(1): 48-51.

[14] Busardo FP, Pichini S. GHB side effects: The need to clearly document drug dose and administration history. J Forensic Leg Med. 
2018; 56: 73-74. PMid:29533209. https://doi.org/10.1016/ j.jflm.2018.03.010

[15] Mmaa EG, Haa AZ, Abozinadah NY, et al. In vivo evaluation of the toxic effect of ethyl acetate extracts of marine antibiotic resistance pseudomonas species derived from the red sea. Appl Biochem Biotechnol. 2018; 184(2): 1-27. PMid:28685240. https: //doi.org/10.1007/s12010-017-2553-x
[16] Chen L, Monteiro T, Wang T, et al. Design of shared unit-dose drug distribution network using multi-level particle swarm optimization. Health Care Manag Sci. 2019; 22(2): 304-317. PMid:29497913. https://doi.org/10.1007/s10729-018-9438-6

[17] Kumar KS, Srinivasan V, Toles RE, et al. High-dose antibiotic therapy is superior to a 3-drug combination of prostanoids and lipid A derivative in protecting irradiated canines. J Radiat Res. 2018; 43(4): 361370. PMid:12674200. https://doi.org/10.1269/jrr.43.361 